\title{
Loss of p53, rather than beta-catenin overexpression, induces survivin-mediated resistance to apoptosis in an esophageal cancer cell line
}

\author{
Elizabeth Chang, BS, James Donahue, MD, Anna Smith, BS, John Hornick, BS, MS, Jaladanki N. Rao, PhD,
} Jian-Ying Wang, MD, PhD, and Richard J. Battafarano, MD, PhD

\begin{abstract}
Objective: Survivin, an important inhibitor of apoptosis, is overexpressed in esophageal cancer and negatively affects survival. The complex regulation of survivin transcription involves enhancement by beta-catenin and repression by p53. The purpose of this study is to test whether inhibition of beta-catenin or overexpression of p53 can decrease survivin expression and render esophageal cancer cells more susceptible to apoptosis.
\end{abstract}

\begin{abstract}
Methods: Studies were performed in normal human esophageal epithelial cells and the human esophageal cancer cell line TE7. Levels of beta-catenin, survivin, and p53 were measured by Western blot. Apoptosis was induced after treatment with camptothecin and measured by release of caspase 3 and morphologic criteria. The roles of survivin and beta-catenin in preventing apoptosis were tested by their silencing with specific small interfering RNA molecules. The effect of $\mathrm{p} 53$ overexpression on survivin promoter activity was measured using a survivin promoter-luciferase reporter construct and by real-time polymerase chain reaction measurement of survivin mRNA levels.
\end{abstract}

Results: Both beta-catenin and survivin are overexpressed in TE7 cells, whereas p53 expression is negligible. TE7 cells demonstrate resistance to camptothecin-induced apoptosis $(P<.01)$. This effect is significantly reduced by inhibition of survivin, but not of beta-catenin $(P<.01)$. Overexpression of p53 in TE7 cells reduces survivin transcription and mRNA levels $(P<.01)$, without reducing survivin protein levels.

Conclusion: Survivin plays a critical role in TE7 cell resistance to camptothecin-induced apoptosis. This effect is not dependent on beta-catenin expression. Overexpression of p53 decreases survivin transcription but does not decrease levels of survivin protein, suggesting posttranscriptional control of survivin expression. (J Thorac Cardiovasc Surg 2010;140:225-32)

There are more than 16,000 new cases of esophageal cancer diagnosed each year in the United States. Despite advances in radiographic and endoscopic detection, chemoradiation therapy, and surgical care, the overall 5-year survival for patients diagnosed with esophageal cancer is $17 \%{ }^{1}$ Most treatment failures result from distant metastatic disease unresponsive to systemic chemotherapy. Identification of the intracellular changes responsible for the development and progression of esophageal cancer provides a potential opportunity for designing rational targeted therapies for the treatment of this lethal disease.

One of the major modes of action of chemotherapy and radiation therapy in the treatment of cancer is the activation of apoptosis. The tumor suppressor gene p53 and its downstream

\footnotetext{
From the Department of Surgery, Division of Thoracic Surgery, University of Maryland School of Medicine, Baltimore, Md. Disclosures: None.

Received for publication June 4, 2009; revisions received Oct 24, 2009; accepted for publication Nov 15, 2009; available ahead of print March 17, 2010.

Address for reprints: Richard J. Battafarano, MD, PhD, 22 South Greene Street, Room N4E35, Baltimore, MD 21201-1544 (E-mail: rbattafarano@smail.umaryland.edu). 0022-5223/\$36.00

Copyright $(c) 2010$ by The American Association for Thoracic Surgery doi:10.1016/j.jtcvs.2009.11.038
}

effector genes p21, mdm-2, and gadd45 are important components in the cellular response to radiation and chemotherapyinduced damage. ${ }^{2,3}$ In addition, a number of members in the Bcl-2 and inhibitor of apoptosis families of proteins (including survivin) have been shown to be overexpressed in cancer cells and demonstrate marked antiapoptotic function. Because the intracellular mechanisms associated with resistance to apoptosis in cancer cells seem to be tissue specific, we sought to specifically investigate the mechanisms responsible for apoptosis resistance in esophageal cancer cells.

Survivin is a member of the inhibitor of apoptosis family of proteins and is an important downstream effector of $\beta$-catenin/T-cell factor (TCF)-mediated transcription. ${ }^{4-7}$ Survivin is normally expressed during the $\mathrm{G} 2 / \mathrm{M}$ phase of the cell cycle and associates with microtubules of the mitotic spindle. Overexpression of survivin functions to overcome this apoptotic checkpoint and favors the aberrant progression of transformed cells through mitosis. ${ }^{8}$ Although survivin is almost undetectable in most normal human adult tissues, it is highly expressed in a number of cancers, including esophageal cancer. ${ }^{9-11}$

The Wnt signaling cascade is important for maintaining epithelial integrity throughout the gastrointestinal tract. Wnt signaling induces growth-promoting (c-myc, cyclin 


\section{Abbreviations and Acronyms \\ nhEso $=$ normal human esophageal \\ PCR = polymerase chain reaction \\ $\mathrm{SEM}=$ standard error of the mean \\ si $\quad=$ small interfering \\ TBS-T $=$ tris-buffered saline/tween 20 \\ $\mathrm{TCF}=\mathrm{T}$-cell factor}

D1) and antiapoptotic (survivin) gene expression through $\beta$ catenin/TCF-mediated transcription. ${ }^{12-14} \mathrm{We}$ previously demonstrated that the Wnt signaling cascade is overexpressed in esophageal cancer cells and that down-regulation of $\beta$-catenin inhibited esophageal cancer cell proliferation and induced spontaneous apoptosis. In addition, inhibition of $\beta$-catenin in esophageal cancer cells was associated with decreased $\beta$-catenin/TCF-mediated transcription and decreased expression of the downstream effectors of Wntsignaling, including the multidrug resistance gene and the transcription factor LEF- $1 .^{15}$

Because Wnt signaling has been demonstrated to induce expression of survivin through $\beta$-catenin/TCF-mediated transcription, we initially hypothesized that this mechanism was responsible for the resistance to apoptosis observed in esophageal cancer cells. The results presented in this study demonstrate that inhibition of survivin expression induced both spontaneous apoptosis and augmented chemotherapy-induced apoptosis in esophageal cancer cells. However, inhibition of $\beta$-catenin expression had no effect on survivin protein expression or spontaneous or chemotherapy-induced apoptosis. Additional experiments demonstrated that overexpression of wild-type p53 protein in esophageal cancer cells resulted in a marked decrease in survivin transcription, but no decrease in survivin protein levels. Taken together, these results are consistent with a model of esophageal cancer cell resistance to apoptosis in which loss of p53 leads to increased survivin gene transcription and additional posttranscriptional regulation facilitates increased survivin protein expression.

\section{MATERIAL AND METHODS \\ Cell Lines and Cell Culture}

The human esophageal cancer cell line TE7 was received as a gift from Dr Nishihira. It was derived from an esophageal adenocarcinoma and characterized in the Cell Resource Center for Biomedical Research, Tohoku University. This cell line was cultured in Roswell Park Memorial Institute (Mediatech Inc, Herndon, Va) supplemented with 10\% heat-inactivated fetal bovine serum, $1 \% \mathrm{~L}$-glutamine, and $1 \%$ penicillin/streptomycin mixture (all from Mediatech Inc), and maintained in a $37{ }^{\circ} \mathrm{C}$ incubator with $5 \% \mathrm{CO}_{2}$ humidified air. Normal human esophageal (nhEso) mucosal cells were derived from human esophageal specimens harvested at the time of donor lung procurement. Esophageal specimens were opened and washed with sterile medium. The mucosa was then removed by careful dissection along the submucosal plane separating the mucosal and muscular layers of the esophagus. This mucosal layer was then cut into small pieces and digested in a collagenase medium to create a single cell suspension. These esophageal mucosa cells were then maintained in BEBM media (Lonza Corporation, Walkersville, Md) supplemented with $20 \%$ heat-inactivated fetal bovine serum and the BEGM BulletKit. Passages 5 to 20 were used for the experiments. Stock cells were maintained in T-150 flasks and incubated at $37^{\circ} \mathrm{C}$ in a humidified atmosphere of $95 \%$ air and $5 \% \mathrm{CO}_{2}$.

\section{Western Blot Analysis}

Whole-cell lysates were prepared using $2 \%$ sodium dodecyl sulfate sample buffer $(250 \mathrm{nM}$ Tris $\cdot \mathrm{HCl}$, pH $6.8,2 \%$ sodium dodecyl sulfate, $20 \%$ glycerol, and 5\% mercaptoethanol), vortexed, and centrifuged $(14,000 \mathrm{~g})$ at $4{ }^{\circ} \mathrm{C}$ for 10 minutes. The supernatants from cell samples were boiled for 5 minutes and then subjected to electrophoresis on $10 \%$ or $15 \%$ sodium dodecyl sulfate-polyacrylamide gel electrophoresis. After transferring proteins onto nitrocellulose filters, the blots were incubated for 1 hour in $5 \%$ nonfat dry milk in $1 \times$ tris-buffered saline/tween 20 (TBS-T). Immunologic evaluation was then performed for 1 hour in $5 \%$ nonfat milk/TBS-T buffer containing the specific antibody against $\beta$-catenin, survivin, and caspase- 3 proteins. The filters were subsequently washed with $1 \times$ TBS-T and incubated for 1 hour with the appropriate secondary antibody conjugated to horseradish peroxidase. After extensive washing with $1 \times$ TBS-T, the immunocomplexes on the filters were reacted for 1 minute with Chemiluminescence Reagent (NEL-100; NEN, Boston, Mass), and exposed to autoradiography film for 10 to 60 seconds.

\section{RNA Interference}

The small interfering (si)RNA specifically targeting the coding region of $\beta$-catenin mRNA (si $\beta$-catenin) was purchased from Dharmacon (Chicago, Ill). The siRNA that was designed to specifically cleave survivin mRNA (siSurvivin) was purchased from Cell Signaling Technology (Boston, Mass). Scrambled control siRNA (C-siRNA), which had no sequence homology to any known genes, was used as the control. For each $100-\mathrm{mm}$ cell culture dish of the si $\beta$-catenin transfection, $20 \mu \mathrm{L}$ of the $10 \mu \mathrm{M}$ stock duplex si $\beta$ catenin or C-siRNA was mixed with $600 \mu \mathrm{L}$ of Opti-MEM medium (Invitrogen, Carlsbad, Calif). For the siSurvivin transfection, $40 \mu \mathrm{L}$ of the $10 \mu \mathrm{M}$ stock duplex siSurvivin or C-siRNA was mixed with $600 \mu \mathrm{L}$ of Opti-MEM medium. The mixture was gently added to a solution containing $10 \mu \mathrm{L}$ of Lipofectamine 2000 (Invitrogen) in $600 \mu \mathrm{L}$ of Opti-MEM. The solution was incubated for 20 minutes at room temperature and gently overlaid onto monolayers of cells in $6 \mathrm{~mL}$ of medium. Cells were harvested for various assays after 72 hours of incubation.

\section{Determination of Apoptosis}

To examine the apoptotic responses of nhEso and TE7 cell lines, we used a camptothecin-induced apoptosis model. After either no manipulation or treatment with indicated siRNA molecules, cells were cultured in serumfree media for 18 hours before the addition of camptothecin $(10 \mu \mathrm{M})$ for 6 hours. Cells were photographed with a Nikon inverted microscope (Tokyo, Japan) before the preparation of whole cell lysates to be used for the detection of caspase- 3 . The number of apoptotic cells was quantified using a morphologic analysis described previously. ${ }^{16}$ Briefly, representative photomicrographs were reviewed by 2 observers. Between 100 and 150 cells were counted, and the number of apoptotic cells were expressed as a fraction of the total number of cells counted.

\section{Reverse Transcription-Polymerase Chain Reaction and Real-Time Polymerase Chain Reaction Analysis}

Total RNA was isolated by using the RNeasy mini kit (QIAGEN, Valencia, CA) and used in reverse transcription and polymerase chain reaction (PCR) amplification reactions. Real-time quantitative PCR was performed using 7500-Fast Real-Time PCR Systems (Applied Biosystems, Foster City, Calif) with specific primers, probes, and software (Applied Biosystems). The levels of survivin mRNA were quantified by quantitative PCR 


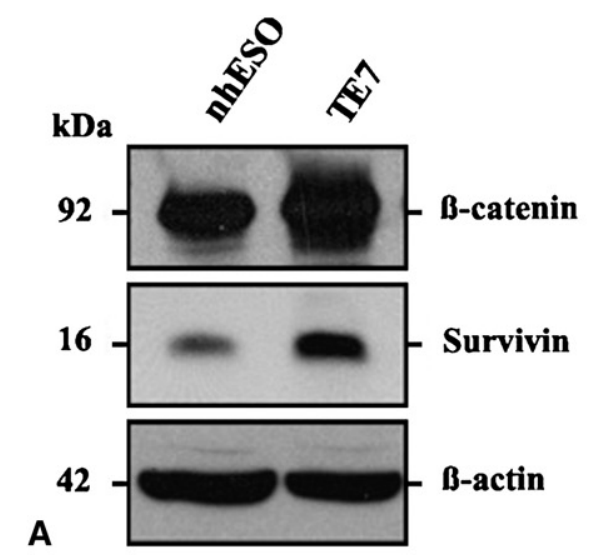

a. nhESO

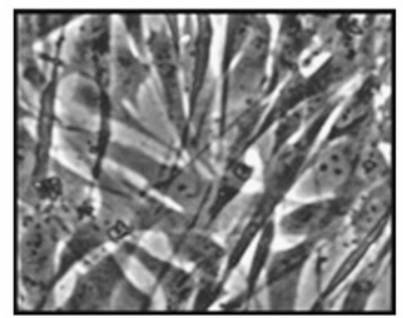

b. TE7

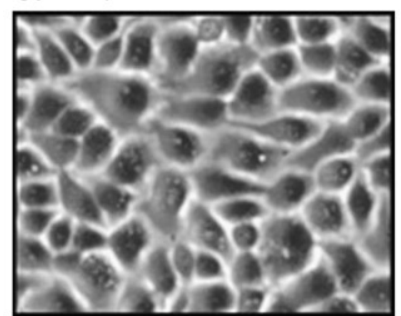

B No-Camptothecin
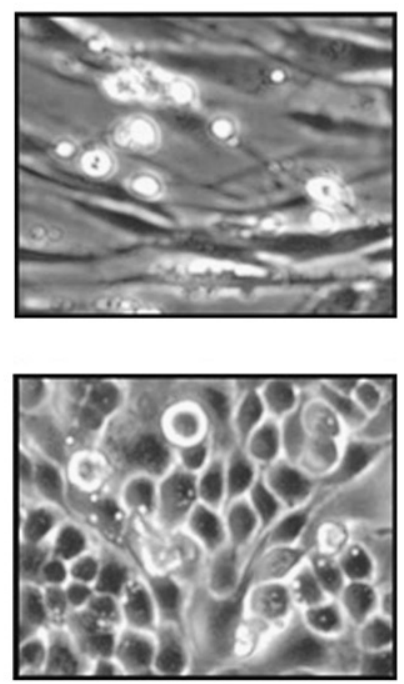

Camptothecin

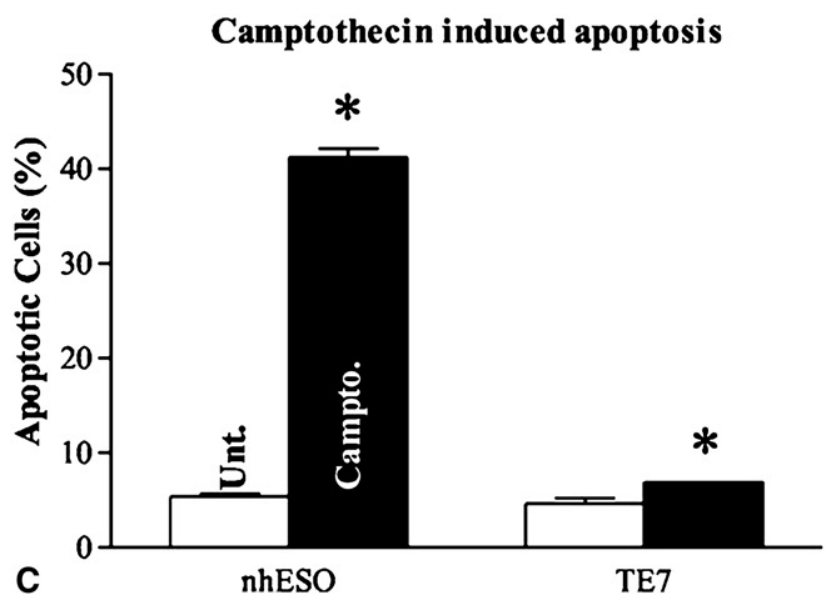

FIGURE 1. TE7 cells overexpress survivin and $\beta$-catenin and are resistant to chemotherapy-induced apoptosis compared with nhEso cells. A, Representative immunoblot of whole cell lysates from nhEso and TE7 cells. Twenty-five milligrams of total protein were applied to each lane, and membranes were hybridized with the antibody specific for survivin $(\sim 16 \mathrm{kDa})$ analysis and expressed as relative to glyceraldehyde-3-phophate dehydrogenase levels.

\section{Reporter Plasmids and Luciferase Assays/Plasmid Construction and Transfection}

Genomic cloning and sequence analysis. Two PCR primers designed from the human survivin gene were used to amplify human genomic DNA. The sequences of the primers were 5'-CTG GCC ATA GAA CCA GAG AAG TGA-3' (sense) and 5'-CCA CCT CTG CCA ACG GGT CCC GCG-3' (antisense). After the resulting PCR product was sequenced to confirm that no mutations were introduced by PCR, the product obtained was subcloned into an expression vector pCR 2.1 by using a TA cloning kit (Invitrogen). One positive clone was identified. Recombinant DNA was prepared using PureYield Plasmid Miniprep System (Promega, Madison, Wis) according to the manufacturer's instructions. Xho I and Hind III digestion of the clone resulted in a $1.1-\mathrm{kb}$ fragment that was then subcloned into the pGL3-Basic Vector (Promega). Sequencing analysis of clone was found to contain $1.1 \mathrm{~kb}$ of the survivin 5' flanking sequence.

Transfection and luciferase assay. nhEso and TE7 cells were seeded in 12-well plates 24 hours before transfection at a density of 6 $\times 10^{4}$ or $3 \times 10^{5}$ cells per well, respectively. At $50 \%$ to $60 \%$ confluence, cells were transfected using Lipofectamine 2000 according to the manufacturer's suggestion. The cells were incubated with Lipofectamine:DNA complexes containing $0.7 \mu \mathrm{L}$ of Lipofectamine, $0.3 \mu \mathrm{g}$ of test plasmid, and $0.15 \mu \mathrm{g}$ of a control plasmid containing the Renilla luciferase reporter gene under the control of a cytomegalovirus enhancer/promoter (Promega). The transfection mixture was replaced by regular growth medium 6 hours later. Measurement of luciferase activity was performed 24 hours after transfection by using the Dual Luciferase Reporter Assay System (Promega) according to the manufacturer's protocol. Briefly, cells were washed twice with PBS solution and solubilized with $100 \mu \mathrm{L}$ of lysis buffer. The lysates were collected, and luciferase assay was determined by using $20-\mu \mathrm{L}$ aliquots of supernatants using a Microtiter Plate Luminometer and Revelation 4.06 Software (Dynex Technologies, Chantilly, Va). The luciferase activity of each construct was compared with that of the promoterless pGL3 basic vector. The levels of luciferase activity from individual constructs were normalized by Renilla-driven luciferase activity in every experiment. Luciferase values are expressed as relative to the highest value obtained in each experiment. Co-transfection assays were carried out in nhEso and TE7 cells using $0.3 \mu \mathrm{g}$ of survivin luciferase reporter gene plasmid, $0.3 \mu \mathrm{g}$ of either an empty expression vector or an overexpression vector encoding the tumor protein p53 (OriGene Technologies Inc, Rockville, Md), in $0.7 \mu \mathrm{L}$ Lipofectamine reagent. The cells were harvested 24 hours after transfection and assayed for luciferase activity as described above.

\section{Statistics}

Values are means \pm standard error from 3 to 6 samples. Autoradiographic and immunoblotting results were repeated 3 times. The significance of the difference between means was determined by analysis of variance.

and $\beta$-catenin ( $\sim 42 \mathrm{kDa})$. After the blot was stripped, actin $(\sim 42 \mathrm{kDa}) \mathrm{im}$ munoblotting was performed as an internal control for equal loading. Three experiments were performed that showed similar results. B, Representative photomicrographs of apoptotic cell death after treatment with (right) or without (left) camptothecin $(10 \mu \mathrm{M})$ for 6 hours. A, nhEso cells. B, TE7 cells. Original magnification $\times 150$. C, Percentage of apoptotic cells as described in B. Values are means \pm standard error (SE) of data from 3 experiments. $* P<.01$ comparing nhEso cells with TE7 cells after treatment with camptothecin. nhESO, Normal human esophageal. 


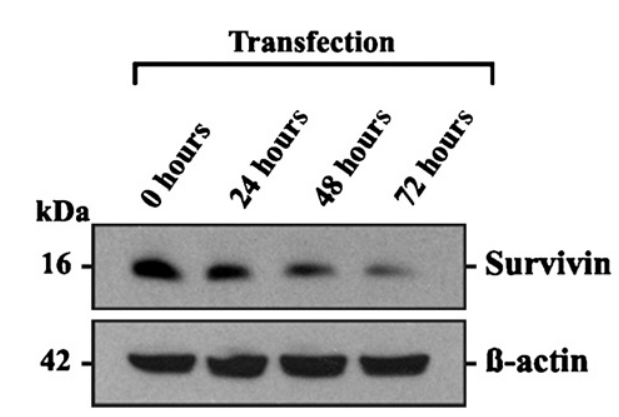

A

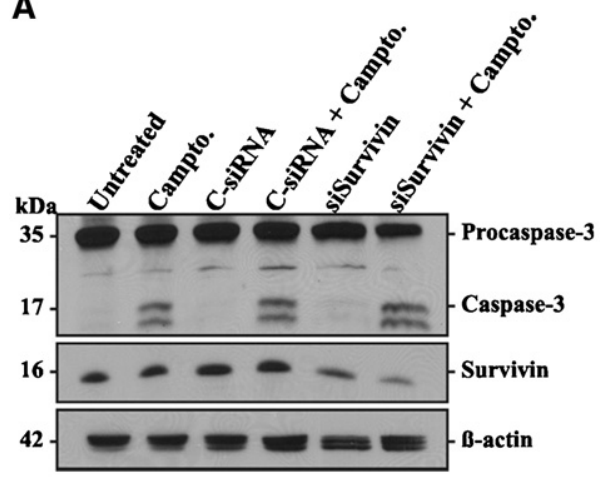

B Camptothecin induced apoptosis

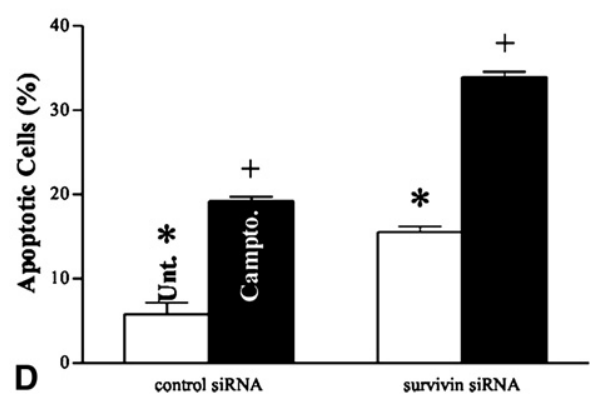

a. Control
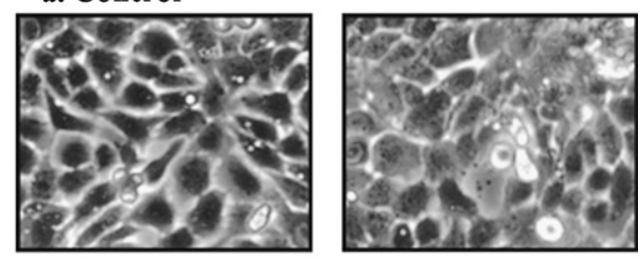

b. Control siRNA
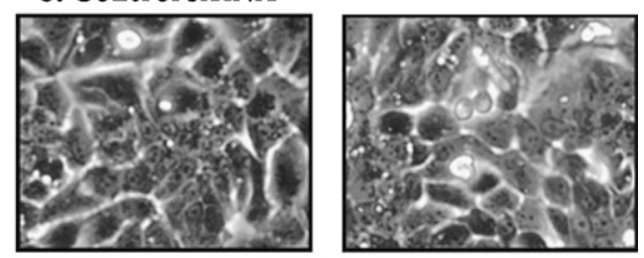

c. Survivin siRNA

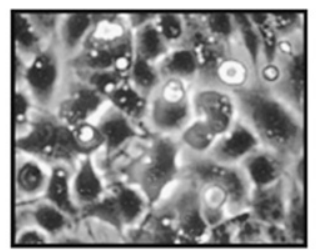

No-Camptothecin

C

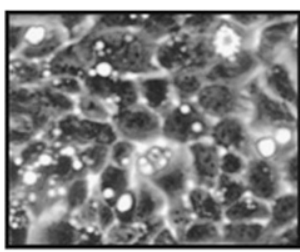

Camptothecin

FIGURE 2. Down-regulation of survivin induces spontaneous apoptosis and enhances chemotherapy-induced apoptosis in TE7 cells. A, Representative immunoblots of survivin expression in TE7 cells 24, 48, and 72 hours after treatment with siSurvivin. B, Representative immunoblots of survivin and caspase-3 protein expression in TE7 cells after transfection with siSurvivin or C-siRNA and subsequent treatment with camptothecin (10 $\mu$ M) for 6 hours. C, Representative photomicrographs of apoptotic cell death in TE7 cells after transfection with siSurvivin or C-siRNA and subsequent treatment with (right) or without (left) camptothecin using the identical conditions described in B. Original magnification $\times 150$. D, Percentage of apoptotic cells as described in C. Values are means $\pm \mathrm{SE}$ of data from 3 experiments. ${ }^{*} P<.01$ comparing cells treated with siSurvivin versus $\mathrm{C}$-siRNA. $+P<.01$ comparing cells treated with siSurvivin and camptothecin versus $\mathrm{C}$-siRNA and camptothecin. nhESO, Normal human esophageal.

\section{RESULTS}

TE7 Cells Overexpress $\beta$-Catenin and Survivin and Are Resistant to Chemotherapy-Induced Apoptosis

To identify the possible mechanism for the resistance to apoptosis observed in esophageal cancer cells, we examined the expression levels of $\beta$-catenin and survivin in TE7 cells relative to nhEso cells. As shown in Figure 1, $A$, both $\beta$-catenin and survivin are overexpressed in TE7 cells compared with nhEso cells. Because resistance to apoptosis is commonly observed in cancer cells, we specifically examined the ability of the chemotherapy agent camptothecin to induce apoptosis in nhEso and TE7 cells. As illustrated in Figure 1,
$B$ and $C$, TE7 cells are significantly more resistant to chemotherapy-induced apoptosis. After exposure to camptothecin, $41.2 \%$ (standard error of the mean [SEM], 0.97) of nhEso cells undergo apoptosis as assessed by morphology compared with only $6.9 \%$ (SEM, 0.05$)$ of TE7 cells $(P<.01)$.

\section{Inhibition of Survivin Expression Induces Spontaneous Apoptosis and Augments Chemotherapy-Induced Apoptosis in TE7 Cells}

To determine whether survivin overexpression was directly responsible for the resistance to apoptosis observed in TE7 cells, survivin expression was inhibited using 


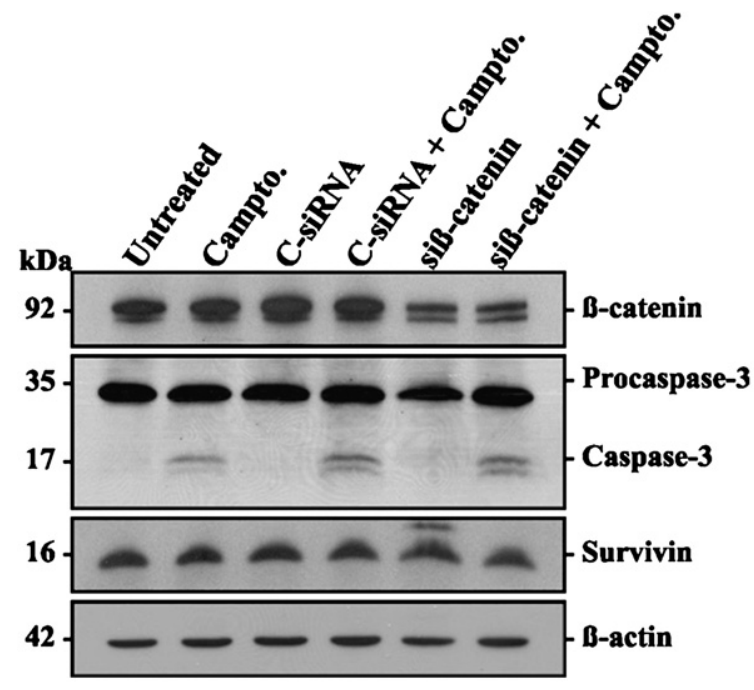

A

Camptothecin induced apoptosis

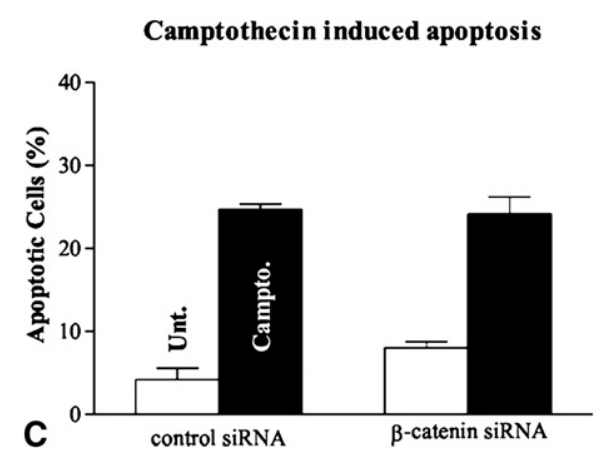

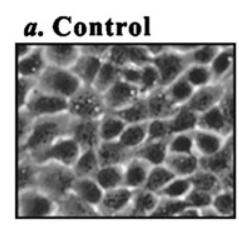

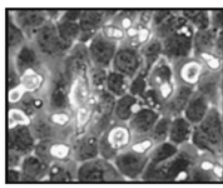

b. Control siRnA
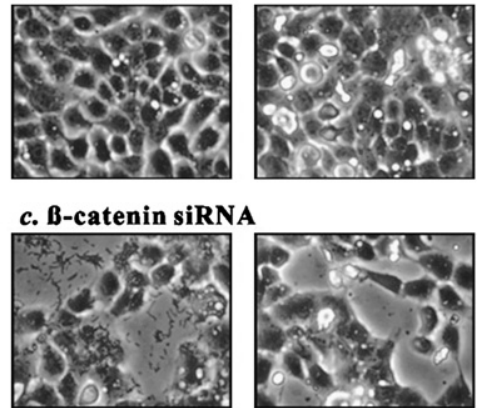

B

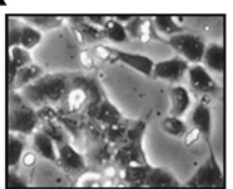

Camptothecin

FIGURE 3. Down-regulation of $\beta$-catenin has no significant effect on survivin levels or apoptosis in TE7 cells. A, Representative immunoblots of $\beta$-catenin, survivin, and caspase-3 protein expression in TE7 cells after transfection with si $\beta$-catenin or C-siRNA. Cells were subsequently treated with camptothecin (10 $\mu \mathrm{M})$ for 6 hours. B, Representative photomicrographs of apoptotic cell death in TE7 cells after transfection with si $\beta$-catenin or C-siRNA and subsequent treatment with $(r i g h t)$ or without (left) camptothecin using the identical conditions described in A. Original magnification $\times 150$. C, Percentage of apoptotic cells as described in $\mathrm{B}$. Values are means $\pm \mathrm{SE}$ of data from 3 experiments. No values exhibited statistically significant differences. $n h E S O$, Normal human esophageal; si, small interfering.

siSurvivin and its effect on spontaneous and chemotherapy induced apoptosis was assessed. As demonstrated in Figure 2, $A$, treatment of TE7 cells with siSurvivin was associated with decreased survivin protein expression with its maximal effect noted from 48 to 72 hours after transient transfection. TE7 cells then were transiently transfected with siSurvivin and subsequently treated with camptothecin. Spontaneous and chemotherapy-induced apoptosis were then assessed by both generation of caspase- 3 from procaspase- 3 and morphology. As seen in Figure 2, B, caspase-3 release was greatest in the cells treated with siSurvivin and subsequently treated with camptothecin. As depicted in Figure 2, $C$ and $D$, treatment of TE7 cells with siSurvivin in combination with camptothecin resulted in a significant increase in apoptosis compared with treatment with C-siRNA. Treatment with siSurvivin resulted in a spontaneous apoptosis rate of $15.6 \%$ (SEM, 0.65) in TE7 cells, versus 5.8\% (SEM, 1.36) after treatment with C-siRNA $(P<.01)$.
Furthermore, after exposure to camptothecin, $33.9 \%$ (SEM, 0.66) of cells treated with siSurvivin underwent apoptosis compared with $19.2 \%$ (SEM, 0.55) of cells treated with $\mathrm{C}$-siRNA $(P<.01)$. These results indicate that survivin overexpression is an important mechanism for the resistance to apoptosis observed in TE7 cells.

\section{Inhibition of $\beta$-Catenin in TE7 Cells Has No Effect on Survivin Expression or Chemotherapy-Induced Apoptosis}

We previously demonstrated that inhibition of $\beta$-catenin expression was associated with an increase in spontaneous apoptosis in TE10 esophageal squamous cancer cells. ${ }^{15}$ Because $\beta$-catenin (in combination with TCF) enhances transcription of the survivin gene, we hypothesized that overexpression of $\beta$-catenin was responsible for survivin overexpression in esophageal cancer cells. In these experiments, $\beta$-catenin expression was inhibited using si $\beta$-catenin and its effect on 
A

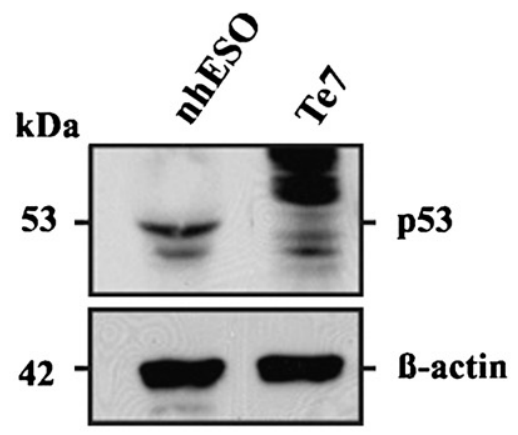

Survivin promoter construct

pGL3 (con)

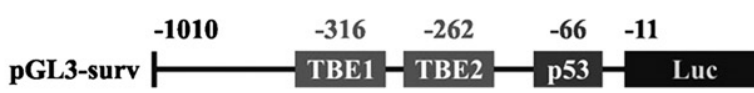

B
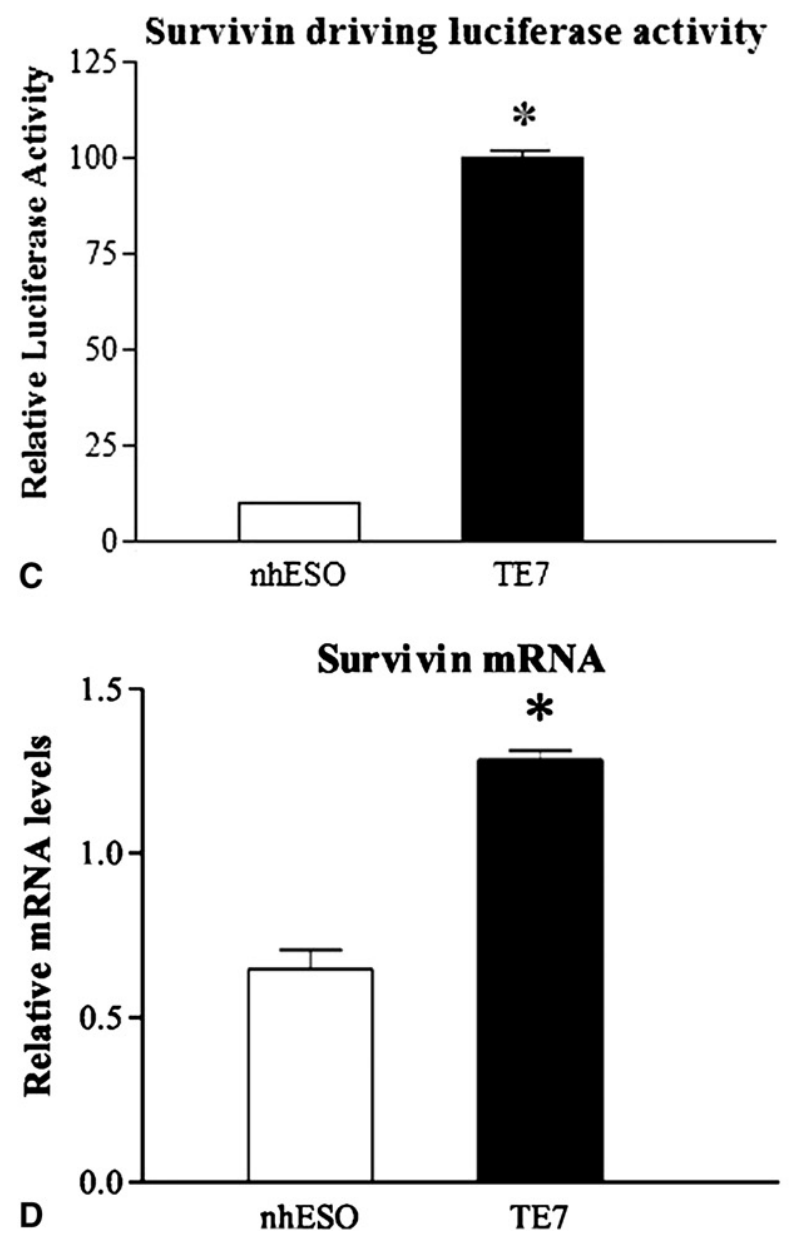

FIGURE 4. TE7 cells exhibit a loss of p53 with resulting increased survivin transcription. A, Representative immunoblots of $\mathrm{p} 53$ protein expression in nhEso and TE7 cells. B, Schematic representation of the survivin promoter-luciferase reporter construct that included 2 TCF- $\beta$-catenin binding survivin expression and spontaneous and chemotherapy-induced apoptosis was assessed. As demonstrated in Figure 3, $A$, treatment of TE7 cells with si $\beta$-catenin had no effect on survivin protein expression. Although caspase-3 release was detectable after exposure to camptothecin, no difference in the number of cells undergoing apoptosis was detected in comparison with C-siRNA (Figure 3, B, C).

\section{TE7 Cells Exhibit Loss of p53 Expression Resulting in Increased Survivin Gene Transcription in Esophageal Cancer Cells}

Although $\beta$-catenin is a known transcriptional activator for the survivin gene and is overexpressed in esophageal cancer cells, we found that it was not directly responsible for survivin overexpression. For this reason, we examined other possible proteins that might regulate survivin expression. p53 is a known repressor of survivin gene transcription that mediates its effect by binding to 533 binding sites in the 5 ' untranslated region of the survivin gene. As demonstrated in Figure 4, A, TE7 cells demonstrate minimal p53 protein expression compared with nhEso cells. To assess the effect of p53 loss on survivin gene transcription, a luciferase reporter construct incorporating the survivin gene promoter was prepared as illustrated in Figure 4, B. In transient transfection experiments shown in Figure 4, $C$, using this luciferase reporter construct, TE7 cells demonstrated 10 times more luciferase activity than nhEso cells $(P=.01)$. Furthermore, as shown by real-time PCR in Figure $4, D$, survivin mRNA levels were twice as high in TE7 cells versus nhEso cells $(P<.01)$.

\section{Overexpression of Wild-type p53 Results in Decreased Transcription of Survivin Gene}

To determine whether loss of $\mathrm{p} 53$ protein was responsible for the survivin overexpression observed in TE7 cells, wildtype human p53 was overexpressed in cultured nhEso cells and in TE7 cells. Overexpression of wild-type human p53 was associated with decreased transcription of the survivin gene in both nhEso cells and TE7 cells (Figure 5, A). In

sites and 1 p53 binding site immediately upstream of the survivin gene. $\mathrm{C}$, Basal activity of the survivin promoter in nhEso and TE7 cells. Cells were transfected with the survivin promoter construct, and levels of the luciferase reporter activity were measured 24 hours later. Results were normalized relative to Renilla-driven luciferase activity and expressed as means $\pm \mathrm{SE}$ of data from 3 separate experiments. Values are expressed as relative to the highest value in each experiment. $* P<.01$ comparing nhEso and TE-7 cells. D, Basal survivin mRNA levels in nhEso and TE7 cells. Total cellular RNA was isolated, and survivin mRNA levels were measured by real-time quantitative PCR analysis. Results are expressed relative to glyceraldehyde-3-phophate dehydrogenase mRNA levels. Values are means \pm SE of data from 3 separate experiments. $* P<.01$ comparing nhEso and TE7 cells. nhESO, Normal human esophageal; si, small interfering. 

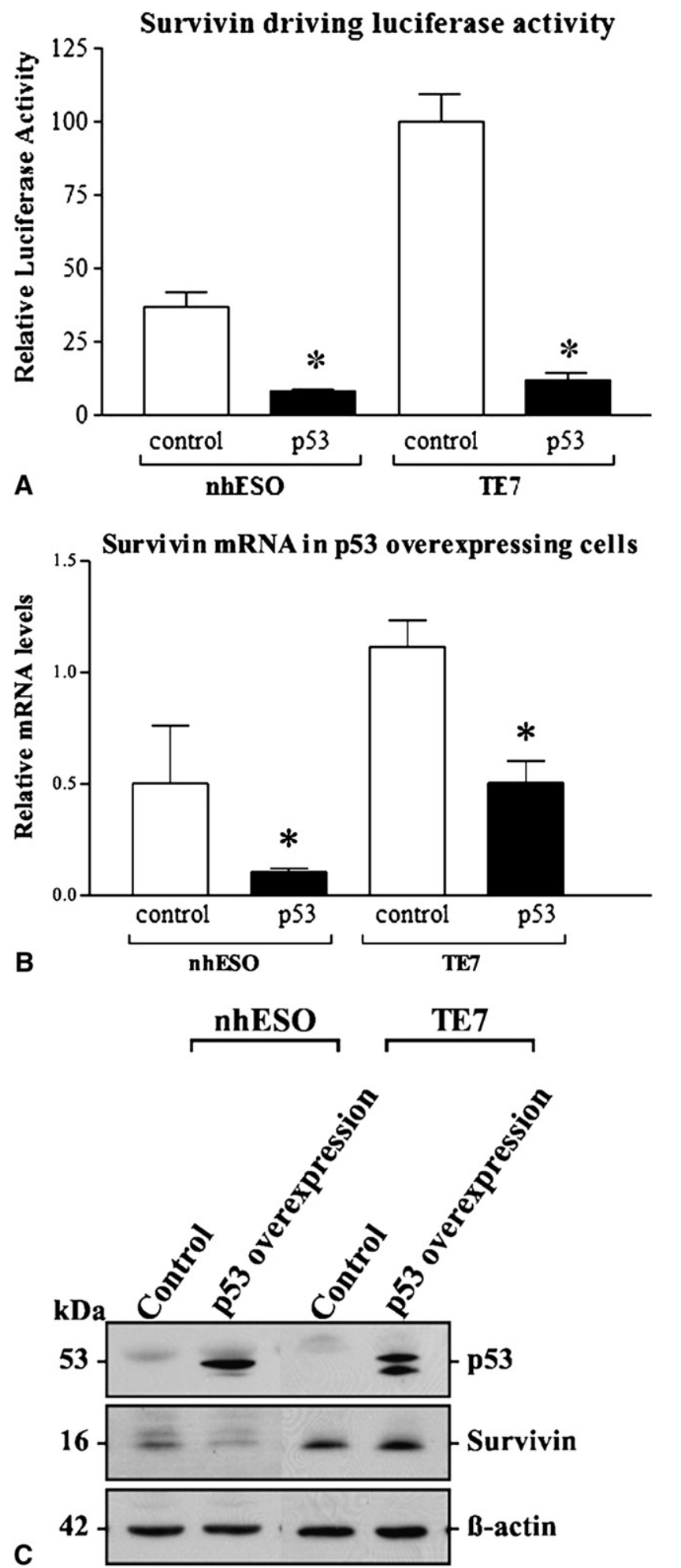

FIGURE 5. Effect of p53 overexpression on survivin promoter activity and survivin mRNA transcription in nhEso and TE7 cells. A, Reporter gene activity in nhEso and TE7 cells after overexpression of p53. Cells were co-transfected with the survivin reporter construct and the expression vector containing human 553 cDNA (p53) or control vector lacking p53 (control) using Lipofectamine (Invitrogen, Carlsbad, Calif). Twenty-four
TE7 cells, luciferase activity decreased by 10 -fold $(P<.01)$ after p53 overexpression. Similarly, as measured by realtime PCR, survivin mRNA levels decreased in both cell types after p53 overexpression (Figure 5, B). In TE7 cells, survivin mRNA levels were reduced by $50 \%$ after p 53 overexpression $(P<.01)$. However, overexpression of wild-type p53 in esophageal cancer cells had no effect on survivin protein levels (Figure 5,C), suggesting a posttranscriptional control point that enhances survivin gene expression.

\section{DISCUSSION}

Overexpression of survivin is observed in many types of human cancer, including esophageal cancer, ${ }^{9-11}$ and is associated with aberrant progression of transformed cells through mitosis. ${ }^{8}$ The results reported in this study clearly demonstrate that overexpression of survivin plays an important role in the resistance to apoptosis observed in TE7 cells. Down-regulation of survivin expression was associated with both spontaneous apoptosis and enhanced susceptibility to chemotherapy-induced apoptosis. Down-regulation of survivin expression in esophageal squamous cancer cells has been shown to inhibit tumor cell growth in vitro and in vivo through increased induction of apoptosis. ${ }^{17}$ To the best of our knowledge, however, this is the first report documenting that survivin overexpression mediates the resistance to apoptosis in esophageal adenocarcinoma cells. Of note, a recent analysis of the TE7 cell line suggests that this line may be a squamous cell cancer and not an adenocarcinoma. ${ }^{18}$

Because we previously demonstrated that down-regulation of $\beta$-catenin expression was associated with decreased

hours later, luciferase reporter activity was measured. Results were normalized relative to Renilla-driven luciferase activity and expressed as means \pm $\mathrm{SE}$ of data from 3 separate experiments. Values are expressed as relative to the highest value in each experiment. $* P<.01$ comparing control versus p53 transfected nhEso and $* P<.01$ comparing control versus p53 transfected TE-7 cells. B, Survivin mRNA levels in nhEso and TE7 cells after p53 overexpression. Cells were co-transfected with the survivin reporter construct and the expression vector containing human p53 cDNA (p53) or control vector lacking p53 (control) using Lipofectamine. Total cellular RNA was isolated, and survivin mRNA levels were measured by realtime quantitative PCR analysis. Results are expressed relative to glyceraldehyde-3-phophate dehydrogenase mRNA levels. Values are means $\pm \mathrm{SE}$ of data from 3 separate experiments. $* P<.01$ comparing control versus p53 transfected nhEso and $* P<.01$ comparing control versus p53 transfected TE-7 cells. C, Effect of p53 overexpression on survivin expression in nhEso and TE7 cells. Cells were transfected with the expression vector containing human p53 cDNA (p53) or control vector lacking p53 (control) using Lipofectamine. Representative immunoblot of whole cell lysates from nhEso and TE7 cells is shown. Twenty-five milligrams of total protein were applied to each lane, and membranes were hybridized with the antibody specific for survivin $(\sim 16 \mathrm{kDa})$ and p53. After the blot was stripped, actin $(\sim 42$ $\mathrm{kDa}$ ) immunoblotting was performed as an internal control for equal loading. Three experiments were performed that showed similar results. nhESO, Normal human esophageal; si, small interfering. 
esophageal cancer cell growth and induction of spontaneous apoptosis, ${ }^{15}$ we initially hypothesized that $\beta$-catenin/TCFmediated transcription of the survivin gene was responsible for the survivin overexpression observed in TE7 cells. However, the results from the experiments presented in this article clearly demonstrate that down-regulation of $\beta$-catenin had no effect on survivin protein expression or spontaneous or chemotherapy-induced apoptosis. Our previous results suggested that down-regulation of $\beta$-catenin expression was associated with the induction of spontaneous apoptosis in esophageal cancer cells. The different result observed in the current study can be explained by the more specific and quantitative assay that was used. Although a caspase-3 release assay was used in both studies, the previous study used a flow cytometry-based assay that was qualitative. The morphologic apoptosis assay used in the current study allowed for a more quantitative analysis of the results.

p53 is known to negatively regulate transcription of the survivin gene. ${ }^{19}$ In the current study, TE7 cells have lost p53 expression and demonstrated increased transcription of the survivin gene. Overexpression of wild-type human p53 in TE7 cells decreased transcription of the survivin gene and decreased survivin mRNA levels. This phenomenon has recently been demonstrated in patients with esophageal cancer. Yang and colleagues ${ }^{20}$ reported that survivin expression in esophageal tumors was higher in tumors with mutant p53 than in those with wild-type p53. In our report, overexpression of wild-type $\mathrm{p} 53$ had no effect on survivin protein expression, suggesting significant posttranscriptional stabilization of either survivin mRNA or the survivin protein itself in TE7 cells.

\section{CONCLUSIONS}

Loss of p53 expression and overexpression of the Wnt signaling pathway ( $\beta$-catenin) have been described in a number of different cancers. However, the downstream effects of these mutations seem to be tissue specific. The results presented in this article demonstrate that loss of p53 contributes to the survivin-mediated resistance to apoptosis observed in TE7 cells and that $\beta$-catenin/TCF-mediated transcription of the survivin gene does not significantly influence survivin expression in these cancer cells. Further analysis with other negative regulators of survivin transcription, such as Kruppel-like factor 4 , will be important to quantify the importance of p53 loss. ${ }^{21}$ Survivin protein levels did not change despite p53 overexpression with a resulting $50 \%$ decrease in survivin promoter activity. This finding prompts speculation that posttranscriptional regulation of survivin expression in TE7 cells seems to stabilize either survivin mRNA or the survivin protein itself. The relative roles of co-factors, such as PLK1, which bind to survivin and enhance its antiapoptotic function, remain to be clarified. ${ }^{22}$ Additional studies identifying additional mechanisms for this posttranscriptional regulation of survivin may yield potential therapeutic targets for reversing survivin-mediated resistance to apoptosis in esophageal cancer cells.

\section{References}

1. Jemal A, Siegel R, Ward E, Hao Y, Xu J, Thun MJ. Cancer statistics, 2009. CA Cancer J Clin. 2009;59:225-49.

2. Sionov RV, Haupt Y. The cellular response to p53: the decision between life and death. Oncogene. 1999;18:6145-57.

3. Vogelstein B, Lane D, Levine AJ. Surfing the p53 network. Nature. 2000;408: 307-10.

4. Li F, Altieri DC. The cancer antiapoptosis mouse survivin gene: characterization of locus and transcriptional requirements of basal and cell cycle-dependent expression. Cancer Res. 1999;59:3143-51.

5. Li F, Altieri DC. Transcriptional analysis of human survivin gene expression. Biochem J. 1999;344:305-11.

6. Zhang T, Otevrel T, Gao Z, et al. Evidence that APC regulates survivin expression: a possible mechanism contributing to the stem cell origin of colon cancer. Cancer Res. 2001;61:8664-7.

7. Kim PJ, Plescia J, Clevers H, Fearon ER, Altieri DC. Survivin and molecular pathogenesis of colorectal cancer. Lancet. 2003;362:205-9.

8. Li F, Ambrosini G, Chu EY, et al. Control of apoptosis and mitotic spindle checkpoint by survivin. Nature. 1998;396:580-4.

9. Kato J, Kuwabara Y, Mitani M, et al. Expression of survivin in esophageal cancer: correlation with the prognosis and response to chemotherapy. Int J Cancer. 2001; 95:92-5.

10. Vallbohmer D, Peters JH, Oh D, et al. Survivin, a potential biomarker in the development of Barrett's adenocarcinoma. Surgery. 2005;138:701-6.

11. Parenti A, Leo G, Porzionato A, Zaninotto G, Rosato A, Ninfo V. Expression of survivin, p53, and caspase 3 in Barrett's esophagus carcinogenesis. Hum Pathol. 2006;37:16-22.

12. He TC, Sparks AB, Rago C, et al. Identification of c-MYC as a target of the APC pathway. Science. 1998;281:1509-12.

13. Mann B, Gelos M, Siedow A, et al. Target genes of beta-catenin-T cell-factor/lymphoid-enhancer-factor signaling in human colorectal carcinomas. Proc Natl Acad Sci U S A. 1999;96:1603-8.

14. Chen S, Guttridge DC, You Z, et al. Wnt-1 signaling inhibits apoptosis by activating beta-catenin/T cell factor-mediated transcription. J Cell Biol. 2001;152: $87-96$.

15. Veeramachaneni NK, Kubokura H, Lin L, et al. Down-regulation of beta catenin inhibits the growth of esophageal carcinoma cells. J Thorac Cardiovasc Surg. 2004; $127: 92-8$.

16. Xiao L, Rao JN, Zou T, et al. Polyamines regulate the stability of activating transcription factor-2 mRNA through RNA-binding protein HuR in intestinal epithelial cells. Mol Biol Cell. 2007;18:4579-90.

17. Wang Y, Zhu H, Quan L, et al. Downregulation of survivin by RNAi inhibits the growth of esophageal carcinoma cells. Cancer Biol Ther. 2005;4:974-8.

18. Boonstra JJ, van der Velden AW, Beerens EC, et al. Mistaken identity of widely used esophageal adenocarcinoma cell line TE-7. Cancer Res. 2007;67:7996-8001.

19. Hoffman WH, Biade S, Zilfou JT, Chen J, Murphy M. Transcriptional repression of the anti-apoptotic survivin gene by wild type p53. J Biol Chem. 2002;277: 3247-57.

20. Yang X, Xiong G, Chen X, et al. Survivin expression in esophageal cancer: correlation with p53 mutations and promoter polymorphism. Dis Esophagus. 2009; 22:223-30.

21. Zhang G, Zhu H, Wang Y, et al. Kruppel-like factor 4 represses transcription of the survivin gene in esophageal cancer cell lines. Biol Chem. 2009;390:463-9.

22. Feng YB, Lin DC, Shi ZZ, et al. Overexpression of PLK1 is associated with poor survival by inhibiting apoptosis via enhancement of survivin level in esophageal squamous cell carcinoma. Int J Cancer. 2009;124:578-88. 